PROCEEDINGS OF THE

AMERICAN MATHEMATICAL SOCIETY

Volume 130, Number 1, Pages 139-143

S 0002-9939(01)06049-X

Article electronically published on May 23, 2001

\title{
THE STRONG RADICAL AND FINITE-DIMENSIONAL IDEALS
}

\author{
BERTRAM YOOD \\ (Communicated by Dale Alspach)
}

\begin{abstract}
Let $A$ be a semi-prime Banach algebra with strong radical $\mathfrak{R}$ (intersection of its two-sided modular maximal ideals). A minimal left or right ideal $K$ of $A$ is infinite-dimensional if and only if $K \subset \mathfrak{R}$. Thus all minimal one-sided ideals in $A$ are finite-dimensional if $A$ is strongly semi-simple.
\end{abstract}

\section{INTRODUCTION}

All ideals considered in this paper are two-sided unless otherwise specified. In his pioneering paper [9, p. 74] Segal called an algebra strongly semi-simple if the intersection $\mathfrak{R}$ of its modular maximal ideals is $(0)$. That intersection $\mathfrak{R}$ is called the strong radical of $A$. For a discussion of $\Re$ see [8, p. 59] and [7, pp. 490-495].

Let $A$ be a semi-prime algebra over a field $\Phi$. In the special case where $A$ is a Banach algebra Smythe [10] showed that, for $x \in A, x A$ is finite-dimensional if and only if $A x$ is finite-dimensional. We show this to be valid for all $A$. Let $\mathfrak{F}$ be the set of $x \in A$ where $x A$ and $A x$ are finite-dimensional. It is shown that $\mathfrak{F}$ is the direct sum of those minimal ideals of $A$ which are finite-dimensional. Then it is shown that $\mathfrak{F} \mathfrak{R}=(0)$ so that $x A$ and $A x$ are infinite-dimensional for all $x \neq 0$ in $\mathfrak{R}$.

We provide more detail in the case of a semi-prime Banach algebra $A$. Let $\mathfrak{S}$ denote the socle of $A$. To say that $\mathfrak{F}=\mathfrak{S}$ is to say that every minimal one-sided ideal in $A$ is finite-dimensional. We find that $\mathfrak{F}=\mathfrak{S}$ if and only if $\mathfrak{R}$ has zero socle. Consequently $\mathfrak{F}=\mathfrak{S}$ if $A$ is strongly semi-simple. More specifically a minimal one-sided ideal $K$ is infinite-dimensional if and only if $K \subset \mathfrak{R}$.

\section{ON FINITE-DIMENSIONALITY}

Let $A$ be a semi-prime algebra over a field $\Phi$. We extend a result of Smythe 9] to show that, for $x \in A, x A$ is finite-dimensional if and only if $A x$ is also finite-dimensional.

2.1. Lemma. Let $W \neq(0)$ be a finite-dimensional right ideal in $A$. There exists an idempotent $p \in W$ where $p v=v$ for all $v \in W$.

Proof. We refer to the proof [5, Th. 1.4.2] of the classical theorem that if $K \neq(0)$ is a right ideal in a semi-prime artinian ring $R$ there exists an idempotent $p$ so that $K=p R$. For Lemma 2.1 finite-dimensionality replaces the descending chain condition in that elegant argument. Only a few minor changes in [5] Th. 1.4.2] provide the proof of this lemma.

Received by the editors January 7, 2000 and, in revised form, June 10, 2000.

2000 Mathematics Subject Classification. Primary 46H10; Secondary 16D25.

(C)2001 American Mathematical Society 
2.2. Theorem. For $x \in A$ we have $x A$ finite-dimensional if and only if $A x$ is finite-dimensional.

Proof. Let $p \neq 0$ be an idempotent in $A$. We show first that $p A$ is finite-dimensional simultaneously with $A p$. Suppose that $A p$ is finite-dimensional. Then so is $p A p$. For each $x \in A$ we define $T_{p x}$ as a mapping of $A p$ into $p A$ by $T_{p x}(y p)=p x y p$. $T_{p x}$ is a linear mapping. The set $\Gamma$ of all linear mappings of $A p$ into $p A p$ is finitedimensional. The mapping $p x \rightarrow T_{p x}$ is a linear mapping of $p A p$ into $\Gamma$. We show that this mapping is injective so that $p A$ is finite-dimensional.

Suppose that $T_{p v}=T_{p w}$. Then $p(v-w) y p=0$ for all $y \in A$. Hence $[p(v-w) A]^{2}=$ (0). As $A$ is a semi-prime algebra we have $p(v-w) A=(0)$ so that, also, $p v=p w$.

Let $\mathfrak{F}_{L}\left(\mathfrak{F}_{R}\right)$ denote the set of all $x \in A$ for which $x A(A x)$ is finite-dimensional. These are ideals in $A$. Let $x \in \mathfrak{F}_{L}, x \neq 0$, and set $V=\{\lambda x: \lambda \in \Phi\}$. Then $K=V+x A$ is a finite-dimensional right ideal. By Lemma 2.1 there is an idempotent $p \neq 0$ so that $K=p A$. Then $A p$ is also finite-dimensional as is $A p x$. But $x \in K$ so that $p x=x$ and $x \in \mathfrak{F}_{R}$.

Henceforth we set $\mathfrak{F}=\mathfrak{F}_{L}=\mathfrak{F}_{R}$. We say that an ideal $K$ is unital if it possesses an identity element $e$ so that $K=e A$. A simple argument shows that $e$ lies in the center of $A$ ([1, Th. 4.4A]). It follows from Lemma 2.1 that any finite-dimensional ideal is unital.

\section{ON MINIMAL IDEALS IN ALGEBRAS}

Let $A$ be a semi-prime algebra over a field $\Phi$. For a subset $K$ of $A$ let $L(K)=$ $\{x \in A: x K=(0)\}$ and $R(K)=\{x \in A: K x=(0)\}$. If $K$ is an ideal in $A$, then $L(K)=R(K)$ by [2, p. 162] and their common value is denoted by $K^{a}$.

3.1. Lemma. Let $W$ be a minimal ideal in $A$. There exists a unique prime ideal $P$ in $A$ such that $P \cap W=(0)$. Also $P=W^{a}$.

Proof. As (0) is the intersection of all the prime ideals in $A$, then there exists a prime ideal $P$ where $P \not \supset W$. Then $P \cap W=P W=(0)$ and $P \subset W^{a}$. Now $W W^{a} \subset P$ so, as $P$ is a prime ideal, $W^{a} \subset P$. Thus $P=W^{a}$. This enforces the uniqueness of $P$.

3.2. Lemma. If the minimal ideal $W$ contains a non-zero idempotent $p$, then the prime ideal $P$ of Lemma 3.1 is a primitive ideal.

Proof. As $p$ fails to be in the radical of $A$, there exists a primitive ideal $P$ which does not contain $p$.

The idempotent $p$ of Lemma 3.2 has the property that, given an ideal $K$ in $A$, either $p \in K$ or $p \in K^{a}$. For if $K \not \supset W$, then $K W=(0)$ and $p \in K^{a}$. Consider an idempotent $e$ such that $e A$ is a minimal right ideal. Given an ideal $K$, either $e \in K$ or $e \in K^{a}$ [12, Lemma 5.1]. It follows that $A e A$ is a minimal ideal of $A$.

3.3. Lemma. The prime ideal $P$ of Lemma 3.1 is a modular maximal ideal if and only if the minimal ideal $W$ is unital.

Proof. Suppose that $W$ is unital with identity element $q$. Then $W=q A$ and $q$ is in the center of $A$. As $A=q A \oplus(1-q) A$ we have $(1-q) A=W^{a}$ and $W^{a}$ is $a$ modular maximal ideal.

Suppose that $W^{a}$ is a modular maximal ideal. Then $W \oplus W^{a}=A$. As $A / W^{a}$ has an identity, we see that $W$ is unital. 
As in [8] by the spectrum $\Sigma$ of $A$ we mean the set of its modular maximal ideals with the hull-kernel topology. The $M \in \Sigma$ which are of the form $W^{a}$ for a minimal ideal $W$ are precisely the $M \in \Sigma$ for which $M^{a} \neq(0)$.

3.4. Lemma. Any $M \in \Sigma$ for which $M^{a} \neq(0)$ is an isolated point of $\Sigma$. If $A$ is strongly semi-simple $M^{a} \neq(0)$ for any isolated point $M$ of $\Sigma$.

Proof. Suppose $M_{0}^{a} \neq(0)$ for $M_{0} \in \Sigma$. Then, by Lemma 3.1, $M_{0}$ is the unique prime ideal of $A$ not containing $M_{0}^{a}$. Hence every $M \in \Sigma, M \neq M_{0}$ contains $M_{0}^{a}$. Thus $M_{0}$ is not in the closure of $\left\{M \in \Sigma: M \neq M_{0}\right\}$.

Suppose that $A$ is strongly semi-simple and that $M_{0}$ is an isolated point of $\Sigma$. Let $Z=\left\{M \in \Sigma: M \neq M_{0}\right\}$; we cannot have $Z=(0)$ for otherwise $M_{0}$ is not an isolated point. However $M_{0} Z=(0)$ so that $M_{0}^{a} \neq(0)$.

In case $A$ is not strongly semi-simple one can have an isolated point $M_{0}$ of $\Sigma$ where $M_{0}^{a}=(0)$. Consider the algebra $A$ of all bounded linear operators on a separable infinite-dimensional Hilbert space $H$. As shown by Calkin [3] the sole modular maximal ideal $M_{0}$ of $A$ is the set of all compact linear operators on $H$. Clearly $M_{0}^{a}=(0)$. We say that the spectrum $\Sigma$ of $A$ is granular if the set of isolated points of $\Sigma$ is dense in $\Sigma$.

3.5. Theorem. Let $\mathfrak{Q}$ be the direct sum of the unital minimal ideals of $A$. A is strongly semi-simple and $\Sigma$ is granular if and only if $\mathfrak{Q}^{a}=(0)$.

Proof. By our earlier remarks $\mathfrak{Q}^{a}=\cap\left\{M \in \Sigma: M^{a} \neq(0)\right\}$. Suppose $\mathfrak{Q}^{a}=(0)$. Clearly $\mathfrak{R}$ as the intersection of all $M \in \Sigma$ is (0). By Lemma 3.4 the set of isolated points of $\Sigma$ is $\left\{M \in \Sigma: M^{a} \neq(0)\right\}$. By the definition of closure in $\Sigma$ we see that $\Sigma$ is granular.

Suppose that $R=(0)$ and $\Sigma$ is granular. Then, by Lemma 3.4, the closure of the set of isolated points of $\Sigma$ is the set of $M \in \Sigma$ containing $\mathfrak{Q}^{a}$. As $\Sigma$ is granular $M \supset \mathfrak{Q}^{a}$ for all $M \in \Sigma$. As $\mathfrak{R}=(0)$ we have $\mathfrak{Q}^{a}=(0)$.

We turn to a discussion of $\mathfrak{F}$. It is readily shown [13, Lemma 1] that $\mathfrak{F}$ is the union of all finite-dimensional ideals of $A$. Each such ideal and therefore $\mathfrak{F}$ is the direct sum of unital finite-dimensional minimal ideals of $A$. In terms of the set $\mathfrak{Q}$ of Theorem 3.5 we have $\mathfrak{F} \subset \mathfrak{Q}$. As $\mathfrak{Q}^{a}$ is the intersection of some $M \in \Sigma$ we see that $\mathfrak{Q}^{a} \supset \mathfrak{R}$.

\subsection{Theorem. $\mathfrak{F} \mathfrak{R}=(0)$.}

Proof. As $\mathfrak{Q Q}^{a}=(0)$ we have $\mathfrak{F} \mathfrak{R}=(0)$.

3.7. Theorem. For every $x \neq 0$ in $\mathfrak{R}$ we have $x A$ and $A x$ infinite-dimensional.

Proof. Let $x \neq 0$ be in $\mathfrak{R}$. As $(\mathfrak{F} \cap \mathfrak{R})^{2}=(0)$ we have $\mathfrak{F} \cap \mathfrak{R}=(0)$. Thus $x \notin \mathfrak{F}$.

As a consequence of Theorem 3.7 every minimal one-sided ideal of $A$ which lies in $\mathfrak{R}$ is infinite-dimensional. In $\S 4$ we show, under the additional requirement that $A$ is a Banach algebra, that every such ideal must be contained in $\mathfrak{R}$.

3.8. Theorem. Let $A$ be a primitive algebra. Then either $\mathfrak{F}=(0)$ or $A$ is finitedimensional and simple.

Proof. Suppose that $\mathfrak{F} \neq(0)$. Then $\mathfrak{F}$ contains a unital minimal ideal $K$ with identity $v$. Then $A=v A \oplus(1-v) A$. By primitivity $(1-v) A=(0)$ so that $A=v A=K$. 
For a particular case let $A$ be the algebra of all bounded linear operators on an infinite-dimensional Banach space. Here $\mathfrak{F}=(0)$.

We let $\mathfrak{S}$ denote the socle of $A$. As pointed out in [13, Lemma 3] we have $\mathfrak{F} \subset \mathfrak{S}$ and $\mathfrak{S}=\mathfrak{F} \oplus\left(\mathfrak{S} \cap \mathfrak{F}^{a}\right)$. If $\mathfrak{F}^{a}=(0)$, then $\mathfrak{S}=\mathfrak{F}$. Also $\mathfrak{R}=(0)$ by Theorem 3.6. This situation occurs in the case of $L^{1}(G)$ for a compact group $G$.

\section{On Banach Algebras}

Henceforth $A$ is a semi-prime Banach algebra. We obtain more detailed information in this case by employing the result [4, Th. 11] that $A$ is finite-dimensional if $A=\mathfrak{S}$.

4.1. Lemma. Let $M \in \Sigma$. Then $M \not \supset \mathfrak{S}$ if and only if $M=K^{a}$ where $K$ is a finite-dimensional minimal ideal of A. Also $M \supset \mathfrak{S}$ if and only if $M \supset \mathfrak{F}$.

Proof. Let $M \in \Sigma, M \not \supset \mathfrak{S}$. There exists a minimal idempotent $e \notin M$. By [12. Lemma 5.1] we get $e \in M^{a}$. Then $M^{a}$ is a simple Banach algebra equal to its socle. Therefore, by [4, Th. 11], $M^{a}=K$ is finite-dimensional and $M=K^{a}$. This argument also shows that if $M \not \supset \mathfrak{S}$, then $M \not \supset \mathfrak{F}$.

Let $W$ be a finite-dimensional minimal ideal. Then $W$ is a unital ideal and also $W^{a} \in \Sigma$ by Lemma 3.3 .

4.2. Theorem. $\mathfrak{F}^{a}=\mathfrak{R}$ if and only if $\Gamma=\{M \in \Sigma: M \not \supset \mathfrak{S}\}$ is dense in $\Sigma$.

Proof. By Lemma 4.1 we see that $\Gamma=\{M \in \Sigma: M \not \supset \mathfrak{F}\}$. Now $\mathfrak{F}$ is the direct sum of unital minimal finite-dimensional minimal ideals and $K^{a} \in \Gamma$ for each such minimal ideal by Lemma 3.3. Therefore $\mathfrak{F}^{a}=\bigcap\{M: M \in \Gamma\}$. Clearly $\mathfrak{F}^{a} \supset \mathfrak{R}$.

Suppose that $\mathfrak{F}^{a}=\mathfrak{R}$. Then any $M \in \Sigma$ contains $\bigcap\{M: M \in \Gamma\}$ so that $\Gamma$ is dense in $\Sigma$. Suppose, conversely, that $\Gamma$ is dense in $\Sigma$. Then our formula above for $\mathfrak{F}^{a}$ shows that every $M \in \Sigma$ contains $\mathfrak{F}^{a}$ so that $\mathfrak{R} \supset \mathfrak{F}^{a}$ and $\mathfrak{R}=\mathfrak{F}^{a}$.

If $A$ is a modular annihilator algebra as defined in [11] or [7, p. 683], then $M \not \supset \mathfrak{S}$ for every $M \in \Sigma$ so that $\mathfrak{F}^{a}=\mathfrak{R}$ in that case.

4.3. Theorem. $\mathfrak{F}^{a} \cap \mathfrak{S}$ is the socle of $\mathfrak{R}$.

Proof. As noted above $\mathfrak{S}=\mathfrak{F} \oplus\left(\mathfrak{F}^{a} \cap \mathfrak{S}\right)$ and $\mathfrak{F} \cap \mathfrak{R}=(0)$. Therefore, as $\mathfrak{R} \cap \mathfrak{S}$ is the socle of $\mathfrak{R}$ by [11 Lemma 3.10], we have $\mathfrak{R} \cap \mathfrak{S} \subset F^{a} \cap \mathfrak{S}$. Suppose that $\mathfrak{F}^{a} \cap \mathfrak{S} \not \subset \mathfrak{R}$. Then there exists $M \in \Sigma$ where $\mathfrak{F}^{a} \cap \mathfrak{S} \not \subset M$. Let $p$ be a minimal idempotent in $\mathfrak{F}^{a} \cap \mathfrak{S}$ where $p \notin M$. As in Lemma 4.1 we have $p \in \mathfrak{F}$ which is impossible. Then $\mathfrak{R} \cap \mathfrak{S}=\mathfrak{F}^{a} \cap \mathfrak{S}$.

We see that $\mathfrak{S}$ is the direct sum of two ideals $\mathfrak{F}$ and $\mathfrak{F}^{a} \cap \mathfrak{S}$ where $x A$ is finitedimensional for all $x \in \mathfrak{F}$ and $x A$ is infinite-dimensional for all $x \neq 0$ in $\mathfrak{F}^{a} \cap \mathfrak{S}$.

4.4. Corollary. A minimal one-sided ideal $K$ in $A$ is infinite-dimensional if and only if $K \subset \mathfrak{R}$.

Proof. If $K \subset \mathfrak{R}$, then $K$ is infinite-dimensional by Theorem 3.7. Let $p A, p^{2}=p$ be an infinite-dimensional right ideal. Then $p \notin F$ so $p \in \mathfrak{F}^{a} \cap \mathfrak{S}$ by [12] Lemma 5.1]. Therefore $p A \subset \mathfrak{R}$ by Theorem 4.3.

If $A$ is strongly semi-simple, then any minimal one-sided ideal is finite-dimensional. Since Segal's day much progress has been made in determining when $L^{1}(G)$ is strongly semisimple. If the closure of the subgroup of inner automorphisms is compact in the Braconnier topology, then $\mathfrak{R}=(0)$. Similarly $\mathfrak{R}=(0)$ if all 
the continuous, topologically irreducible, unitary representations of $G$ are finitedimensional. These two classes are denoted by $[F I A]^{-}$and [Moore]. We refer to the work of T. W. Palmer [6].

Corollary 4.4 shows that $\mathfrak{F}=(0)$ if and only if $\mathfrak{R} \supset \mathfrak{S}$. By [4, Th. 11] any simple infinite-dimensional Banach algebra $A$ has $\mathfrak{F}=(0)$. If that Banach algebra $A$ has no identity, then also $\Sigma$ is the empty set and $A=\mathfrak{R}$. An example with these properties is an infinite-dimensional simple $H^{*}$-algebra [8, p. 275]. Another example with $\mathfrak{F}=(0)$ and $\Sigma$ empty is the Banach algebra of all compact linear operators on an infinite-dimensional Banach space. Also (see Theorem 4.2) $\mathfrak{F}=(0)$ if and only if $\Sigma$ is empty in the case of a modular annihilator Banach algebra $A$.

\section{REFERENCES}

1. E. Artin, C. J. Nesbitt and R. M. Thrall, Rings with minimum condition, Univ. of Michigan Press, Ann Arbor, Mich., 1944. MR 6:33e

2. F. F. Bonsall and J. Duncan, Complete normed algebras, Springer, New York, 1973. MR 54:11013

3. J. W. Calkin, Two-sided ideals and congruences in the ring of bounded linear operators in Hilbert space, Ann. of Math. 42 (1941), 839-873. MR 3:208c

4. L. Dalla, S. Giotopoulos and N. Katseli, The socle and finite-dimensionality of a semi-prime Banach algebra, Studia Math. 92 (1989), 201-204. MR 90f:46079

5. I. N. Herstein, Noncommutative rings, Carus Math. Monographs 15, Math. Assoc. America, 1968. MR 37:2790

6. T. W. Palmer, Classes of non-abelian, non-compact, locally compact groups, Rocky Mountain J. Math. 8 (1978), 683-741. MR 81j:22003

7. T. W. Palmer, Banach algebras and the general theory of $*$-algebras, vol. I, Cambridge LL. Press, 1994. MR 95c:46002

8. C. E. Rickart, General theory of Banach algebras, Van Nostrand, Princeton, 1960. MR 22:5903

9. I. E. Segal, The group algebra of a locally compact group, Trans. Amer. Math. Soc. 61 (1947), 69-105. MR 8:438c

10. M. R. F. Smythe, On problems of Olubummo and Alexander, Proc. Royal Irish Acad. 80A (1980), 69-74.

11. B. Yood, Ideals in topological rings, Can. J. Math. 16 (1964), 28-45. MR 28:1505

12. Closed prime ideals in topological rings, Proc. London Math. Soc. (3) 24 (1972), 307-323. MR 45:7475

13. _ Finite-dimensional ideals in Banach algebras, Colloq. Math. 63 (1992), 295-301. MR 93m:46052

Department of Mathematics, Pennsylvania State University, University Park, PennSYLVANIA 16802 